\title{
Hsp90 $\beta$ promoted endothelial cell-dependent tumor angiogenesis in hepatocellular carcinoma
}

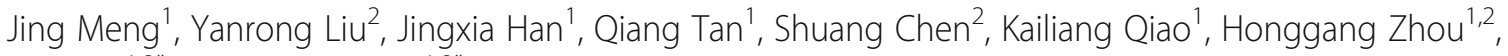
Tao Sun ${ }^{1,2^{*}}$ and Cheng Yang ${ }^{1,2^{*}}$

\begin{abstract}
Background: Vascular endothelial growth factor receptors (VEGFRs) are the major receptors involved in endothelial cell-dependent tumor angiogenesis. There are studies account for the effects of Hsp90 on angiogenesis, but the role and mechanism of Hsp90 3 isoforms and NVP-BEP800, a specific inhibitor of Hsp90ß, in tumor angiogenesis is rarely mentioned.

Methods: Immunohistochemistry and statistical analysis was used to evaluate the correlation between Hsp90 $\beta$ expression, CD31 endothelial cell-dependent vessel density, and VEGFRs expression in tissue samples of 96 HCCs. Kaplan-Meier survival analysis and COX proportional hazards analysis the relation of Hsp90ß and prognosis. HUVEC cells were transfected with Hsp90ß or treated with NVP-BEP800, and then cell proliferation, migration, invasion and tube formation were investigated. The VEGFR1 and VEGFR2 expression was determined by Western blot and immunofluorescence. The VEGFR1 and VEGFR2 promoter activities were detected by dual luciferase report system. In vivo, the angiogenesis promotion of Hsp90 3 and anti-angiogenesis efficacy of NVP-BEP800 was tested in HCC xenograft models. Histological analysis was performed on tumor samples to evaluate Hsp90ß, VEGFRs expression and MVD.
\end{abstract}

Results: This study investigated the correlation between Hsp90ß expression and CD31+ endothelial cell-dependent vessel density. Hsp90ß promoted VEGFRs expression by increasing their promoter activities. The proliferation, migration, invasion, and tube formation activities of human endothelial cells significantly increased when Hsp90 $\beta$ was overexpressed. NVP-BEP800 down-regulated VEGFRs expression to significantly reduce tubular differentiation, as well as endothelial cell proliferation, migration, and invasion. Furthermore, NVP-BEP800 decreased VEGFR1 and VEGFR2 promoter activities. In vivo, Hsp90ß promoted VEGFRs and CD31 expression in human hepatocellular carcinoma tumor xenografts and was associated with increased tumor microvessel density. After 18 days of treatment with $30 \mathrm{mg} / \mathrm{kg} /$ day NVP-BEP800, VEGFRs and CD31 expression significantly decreased.

Conclusion: Hsp90ß induced endothelial cell-dependent tumor angiogenesis by activating VEGFRs transcription. NVP-BEP800 has potential as a therapeutic strategy for inhibiting tumor angiogenesis by decreasing endothelial cell progression and metastasis. It can help develop a therapeutic strategy for tumor treatment through the inhibition of endothelial cell progression and metastasis.

Keywords: Hsp90ß, Angiogenesis, NVP-BEP800, Endothelial cell, Hepatocellular carcinoma

\footnotetext{
* Correspondence: sunrockmia@hotmail.com; cheng.yang@nankai.edu.cn

${ }^{1}$ State Key Laboratory of Medicinal Chemical Biology and College of

Pharmacy, Nankai University, Tianjin 300350, China

Full list of author information is available at the end of the article
}

(c) The Author(s). 2017 Open Access This article is distributed under the terms of the Creative Commons Attribution 4.0 International License (http://creativecommons.org/licenses/by/4.0/), which permits unrestricted use, distribution, and reproduction in any medium, provided you give appropriate credit to the original author(s) and the source, provide a link to the Creative Commons license, and indicate if changes were made. The Creative Commons Public Domain Dedication waiver (http://creativecommons.org/publicdomain/zero/1.0/) applies to the data made available in this article, unless otherwise stated. 


\section{Background}

Tumor initiation and malignant evolution are closely related to angiogenesis $[1,2]$. A physiological or pathological neovascularization process is necessary to initiate tumor tissue ischemia, growth, or metastasis [3]. In angiogenesis, endothelial cell proliferation and migration cause new capillaries to develop from preexisting capillaries [4]. In addition, tumor cells, pericytes, immune cells, hematopoietic cells, circulating endothelial progenitor cells, and various types of molecules are also involved in the complicated process of angiogenesis [5]. Angiogenesis involves complex signaling pathways and many key regulatory factors, such as vascular endothelial growth factor (VEGF)/vascular endothelial growth factor receptor (VEGFR) [6-8], angiopoietins, integrins, neuropilin-1, thrombospondin-1, vasohibin, and $\mathrm{NOTCH} /$ delta-like protein 4 pathway [5]. Among these pathways, the VEGF/VEGFR system of endothelial cells is the classical pathway used in blood vessel formation $[9,10]$. The VEGF receptor family includes VEGFR1, VEGFR2, and VEGFR3 [11]. The high affinity of VEGF and VEGFR1, VEGFR2 induce proliferation, migration and differentiation of vascular endothelial cells [12]. Activated endothelial cells degrade the extracellular matrix, subsequently form tubular structures, and recruite supporting cells to form stable vessels $[1,13,14]$. Therefore, VEGF/VEGFR is an crucial pathway in angiogenesis of tumor.

Heat shock protein 90 (Hsp90) is a molecular chaperone that is induced in response to cellular stress. Hsp90 stabilizes client proteins involved in the cell cycle, proliferation, migration, and apoptosis [15]. The Hsp90 family has four major members: Hsp90 $\alpha$, Hsp90 $\beta$, GRP94, and Hsp75. Evidence suggest that Hsp90 is important in regulating tumor angiogenesis. Hsp90, as a key protein chaperone, plays an important role in the PI-3 K/AKT/mTOR complex of the PI-3 K/AKT signaling pathway, which promotes endothelial cell survival $[16,17]$. VEGFR2 is also reported to be a client protein of Hsp90, which can form a complex with human umbilical vein endothelial cells (HUVEC) [18]. Furthermore, the Hsp90 inhibitor suppresses the expression of VEGFRs and VEGFR complexes, such as integrins [19], focal adhesion kinase (FAK), and neuropilin coreceptors [5]. Although most studies account for the effects of Hsp $90 \alpha$ isoforms on angiogenesis, the role and mechanism of Hsp90 $\beta$ isoforms in tumor angiogenesis is rarely mentioned.

We have previously established that Hsp90 $\beta$ is associated with the tumor malignancy of hepatocellular carcinomas (HCCs). HCC is a highly vascular tumor, thus providing an attractive target for the development of new anticancer drugs is necessary [20]. In the present study, we evaluate whether Hsp90 $\beta$ expression increased in HCCs with a high degree of malignancy. Results showed that there was an obvious relationship between Hsp90 $\beta$ expression and endothelial cell-dependent vessel density. Hsp90 $\beta$ increased VEGFRs expression by activating their promoters and enhancing endothelial cell proliferation, migration, and tubular structure formation. NVP-BEP800, an Hsp90 $\beta$ inhibitor, decreased VEGFRs expression and inhibited HCC tumor angiogenesis. Therefore, NVPBEP800 has potential as a therapeutic strategy for inhibiting tumor angiogenesis by decreasing endothelial cell progression and metastasis.

\section{Methods}

\section{Cell culture and transfections}

HUVECs and PLC-PRF-5 cells were obtained from the Cell Bank of Shanghai Institute for Biological Sciences (Shanghai, China). HUVEC cells were cultured in medium 199 (M-199) containing endothelial cell growth supplement, 10\% fetal bovine serum (FBS), and 1\% antibiotics $(100 \mathrm{U} / \mathrm{mL}$ penicillin and $10 \mu \mathrm{g} / \mathrm{mL}$ streptomycin). PLC-PRF-5 cells were cultured in Roswell Park Memorial Institute 1640 medium supplemented with $10 \%$ FBS and penicillin-streptomycin solution. All cells were maintained at $37{ }^{\circ} \mathrm{C}$ in a $5 \% \mathrm{CO}_{2}$ incubator. All the plasmids were transfected into cells by Lipofectamine 2000 (Invitrogen, USA).

\section{Luciferase activity assays}

HUVECs were seeded in 96-well plates. After $24 \mathrm{~h}$, the plasmids, namely, pEZX-PG04-VEGFR1 and pEZXPG04-VEGFR2 (FulenGene, China), were either transfected separately into cells, co-transfected with pcDNA3Hsp90 $\beta$ or pRNAT-U6-siHsp90 $\beta$, or incubated with NVPBEP800 (Selleck, USA) for $48 \mathrm{~h}$. Gaussia and secreted alkaline phosphatase (SEAP) luciferase activities were consecutively measured by a dual-luciferase reporter assay system (FulenGene, China) after $48 \mathrm{~h}$ of transfection. Gaussia luciferase activity was normalized to SEAP activity.

\section{Cell invasion assays}

HUVECs in serum-free medium were seeded onto a chamber coated with Matrigel (BD, USA) and inserted into the wells of a 24-well plate. FBS was added to a 24well plate located below the chamber to serve as a chemoattractant. After $24 \mathrm{~h}$, invasive cells located on the lower surface of the chamber were stained with $0.1 \%$ crystal violet and counted.

\section{Wound healing assay}

HUVECs were seeded at $80 \%$ to $90 \%$ confluence in 24 well plates. An area of cells was removed with a pipette tip drawn across the center of each well. Cells were washed thrice followed by treatment with FBS-free medium containing DMSO or $2 \mu \mathrm{M}$ NVP-BEP800. The speed of wound closure was monitored after 16, 32, and 
$48 \mathrm{~h}$ by comparing the wound distance ratio at $0 \mathrm{~h}$. This experiment was independently repeated thrice.

\section{Tube formation assay}

HUVECs were seeded onto a 48-well plate coated with Matrigel (BD, USA) followed by treatment with a medium containing DMSO or $2 \mu \mathrm{M}$ NVP-BEP800. Peak tube formation was observed at $12 \mathrm{~h}$ post-treatment. Four random fields were observed under a microscope. The number of tubes for each treatment was quantified. This experiment was independently repeated thrice.

\section{Western blot analysis}

Cells were washed with phosphate-buffered saline (PBS) and lysed in ice-cold lysis buffer containing protease inhibitor cocktail (Sigma) for $30 \mathrm{~min}$. Lysates were separated by electrophoresis and transferred onto polyvinylidene difluoride membranes (Millipore; Bedford, MA, USA). The membranes were blocked and incubated with the primary antibodies of Hsp90 $\beta$, VEGFR1, and VEGFR2 (CST, USA). The membranes were then incubated with a goat anti-rabbit IgG-HRP secondary antibody (Thermo Scientific, USA). GAPDH was used as the loading control. Protein expression was assessed with enhanced chemiluminescent substrate (Millipore, USA) and by exposure to chemiluminescent film.

\section{Immunofluorescence}

HUVECs were fixed with $3.7 \%$ formaldehyde in PBS for $5 \mathrm{~min}$ at room temperature and blocked with $1 \%$ bovine serum albumin for $30 \mathrm{~min}$. The cells were incubated overnight at $4{ }^{\circ} \mathrm{C}$ with VEGFR1 antibody (1:200; CST, USA) or VEGFR2 antibody (1:200, CST, USA), and then incubated with FITC- and TRITC-labeled secondary antibodies (Earthox LLC, USA) for $1 \mathrm{~h}$ at room temperature. Each step was followed by two 5-min washes in PBS. The prepared specimens were counterstained with DAPI (Southern Biotechnology Associates Inc., USA) for 2 min and observed with a confocal microscope (Nikon, Japan).

\section{Immunohistochemistry assay and analysis}

Paraffin sections of human samples and tumor tissue of mice were deparaffinized in xylene and rehydrated in a series of graded alcohols and antigen was retrieved in $0.01 \mathrm{M}$ sodium citrate buffer. Samples were incubated with $3 \% \mathrm{H}_{2} \mathrm{O}_{2}$ for 10 min and then blocked in $1 \%$ bovine serum albumin in PBS for $1 \mathrm{~h}$. The slides were incubated overnight at $4{ }^{\circ} \mathrm{C}$ with Hsp90ß, VEGFR1, VEGFR2, CD31 and Ki67 primary antibody. Using the known positive section as the positive criteria, PBS displaces primary antibody as negative criteria. The secondary antibody was followed added with using HRP-Polymer anti-Mouse/Rabbit IHC Kit (Maixin Biotech, China) for $1 \mathrm{~h}$ at room temperature. Then, samples were developed with diaminobezidine (Vector Laboratories) reagent and counterstained with hematoxylin and mounted with permount [21].

ImageJ software was used to quantify the MVD based on CD31 staining. The percentage of positively stained area was calculated by using a color deconvolution for separating the staining components (diaminobezidine and hematoxylin) in at least six fields per each sections [21]. The results were presented as percentage of treated group compared to control one. All sections were analyzed and evaluated independently by two pathologists (T.S. and Y.R.L.) at double blinded, and the results were reconfirmed once inconsistent.

\section{Xenograft tumor model}

Approximately $1 \times 10^{6}$ PLC or PLC-Hsp90 $\beta$ cells were injected subcutaneously into the middorsal region of each BALB/c nude mouse. Each group (Control, Hsp90 $\beta$ and NVP-BEP800 group) consisted of eight mice. Tumor-bearing mice began to receive treatment at the time point when the average tumor size reached 100$200 \mathrm{~mm}^{3}$. NVP-BEP800 was formulated as a suspension in $0.5 \%$ methylcellulose and was given at the dose of $30 \mathrm{mg} / \mathrm{kg}$ per day by intragastric administration for 18 days [22]. Solvent buffer at the same volume was used in control and Hsp90 $\beta$ groups. Tumor sizes were measured every 2 days using a caliper and calculated according to a standard formula (length $\times$ width $^{2} \times 0.52$ ) as previously described [23].

\section{Patient samples}

HCC tissue microarrays containing 96 cases were purchased from US Biomax for IHC. Tissue blocks of the tissue array were collected within 5 years. Each single tissue spot on every array slide was individually examined by certified pathologists according to the WHO published standardizations for diagnosis, classification and pathological grade. Both the hospital and the individual consented for each specimen from clinical patients to be included. All tissues were collected under the highest ethical standards; the donor was completely informed and provided consent. All human tissues are collected under HIPPA-approved protocols.

\section{Statistical analysis}

Statistical analyses were performed with GraphPad Prism 6 and SPSS v. 19. Statistically significant differences were calculated by student's $t$-test, Wilcoxon rank-sum test, Mann-Whitney $U$ test, and Pearson's correlation as appropriate. Values of $p<0.05$ were considered significant. 


\section{Results}

\section{Hsp90 $\beta$ was associated with HCC angiogenesis}

Our previous study confirmed that Hsp90 $\beta$ was closely related to the metastasis and recurrence of HCC. Based on the immunohistochemical (IHC) analysis of 96 HCC cases, the expression level of Hsp90 $\beta$ and angiogenesis were higher in HCC with a high degree of malignancy than in HCC with a low degree of malignancy (Fig. 1a). The expression of CD31, a surface marker of neovascular endothelial cells, was detected by immunohistochemical staining of HCC tissues in different stages. Then, microvessel density (MVD) was calculated (Fig. 1b). Pearson correlation and linear regression anaysis showed that the levels of steady state expression of the mRNA of Hsp90 $\beta$ and CD31 was positively correlated (Additional file $1 \mathrm{Fig}$. S1a). We further analyzed the correlation between Hsp90 $\beta$ expression and MVD. The results showed that Hsp90 $\beta$ was positively correlated with MVD in $\mathrm{HCC}(P=0.027)$ (Fig. 1c).

\section{Hsp90 $\beta$ indicated poor prognosis}

Kaplan-Meier survival analysis revealed that patients with high levels of Hsp90 $\beta$ expression had significantly lower overall survival (OS) compared with those with low Hsp90 $\beta$ expression $(P=0.000)$ (Fig. 2a). Cox proportional hazards model analysis was performed on the basis of clinicopathological factors, Hsp90 $\beta$ expression (high/low), and MVD presence (positive/negative). Multivariate analysis showed that high Hsp90 $\beta$ expression, MVD, advanced clinical stage, and histological differentiation were indicators of poor prognosis $(P=0.018$, 0.047, 0.022, and 0.009, respectively) (Fig. 2b).

\section{Correlation between Hsp90 $\beta$ and EGFRs}

We analyzed 96 liver cancer cases and found that the expression levels of Hsp90ß, VEGFR1, and VEGFR2 in liver tumor tissues were correlated with the pathological grade and clinical stage of the tumor (Table 1). Immunohistochemical staining showed that HCC patients with high Hsp90 $\beta$ expression levels also highly expressed VEGFR1 and VEGFR2. Additionally, VEGFR1 and VEGFR2 expression levels were higher in the endothelial cells surrounding vessels (Fig. 3a). Correlation analysis showed that Hsp90 $\beta$ was associated with VEGFR1 and VEGFR2 expression $(P=0.022$ and $P=0.035$, respectively) (Fig. $3 \mathrm{~b}$ ). The correlation studies of Hsp90 $\beta$ and VEGFR1, VEGFR2 mRNA levels were consistent with those of IHC evaluation (Additional file 1 Fig. S1b, c).

\section{Hsp90 $\beta$ stimulated endotheliocytes to proliferate and accelerate neovascularization depending on VEGFR expression}

VEGFRs are major receptors on endothelial cells that are involved in multiple signaling pathways, including the induction of mitogenesis, migration, invasion, and differentiation in neoangiogenesis [24]. Sanderson et al. [18] reported that VEGFR2 is a client protein of Hsp90 and they can form a complex in HUVECs to promote endothelial cell migration; they also reported that VEGFR2 deletion mutants are unable to associate with Hsp90 [19, 25]. To elucidate the underlying mechanism of Hsp90 $\beta$ in inducing angiogenesis, we evaluated the effects of Hsp90 $\beta$ on VEGFRs expression. Our results showed that as with VEGF treated HUVEC cells, VEGFR1 and VEGFR2 expression levels also increased after Hsp90 $\beta$ overexpression (Fig. 4a). This result was validated by immunofluorescence staining (Fig. 4b). Whereas, VEGFR1 and VEGFR2 expression levels decreased after Hsp90 $\beta$ knocked down. When the HUVEC cells simultaneously treated with siHsp90 $\beta$ and VEGF, VEGFR1 and VEGFR2 expression levels increased

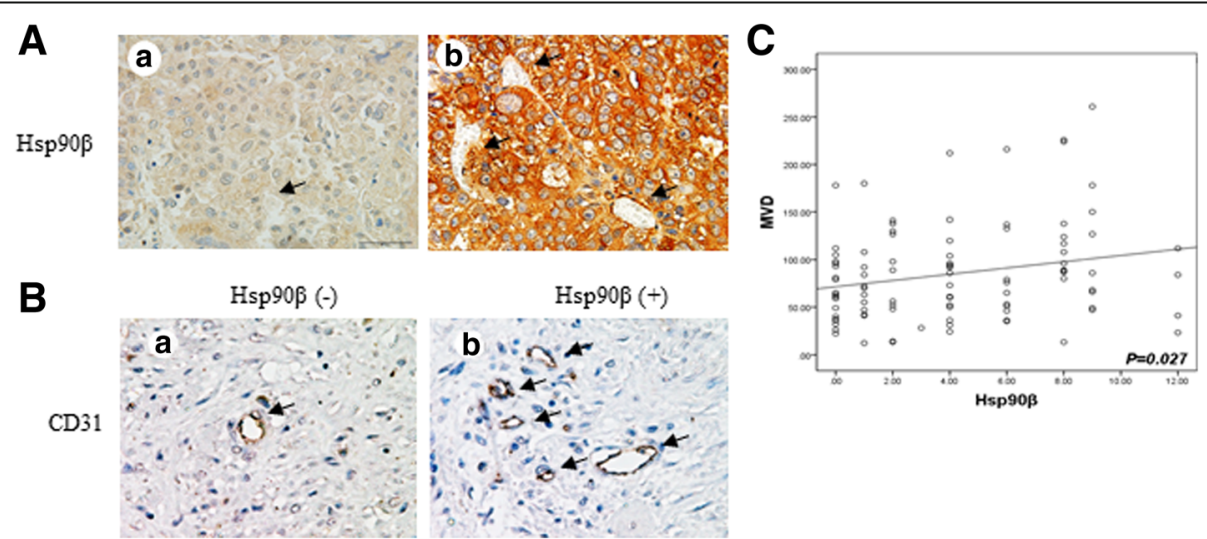

Fig. 1 Correlation between Hsp90 $\beta$ and MVD. a Representative images of IHC staining for Hsp90 $\beta$ in tissue microarrays of human HCC at different stages (left, stage l; right, stage IV) showed weak expression in primary cancer tissues (stage I) and strong expression in metastatic tissues (stage IV). Black arrows point to microvessels. b MVD measured by immunostaining for CD31 in HCC tissue microarrays. MVD was higher in the Hsp90 3 -positive group than in the Hsp90ß-negative group. Black arrows point to microvessels. c MVD and Hsp90ß-positive stainings were quantified and the correlation was analyzed with Spearman correlation method (correlation coefficient: $R=0.226, P=0.027$ ) 

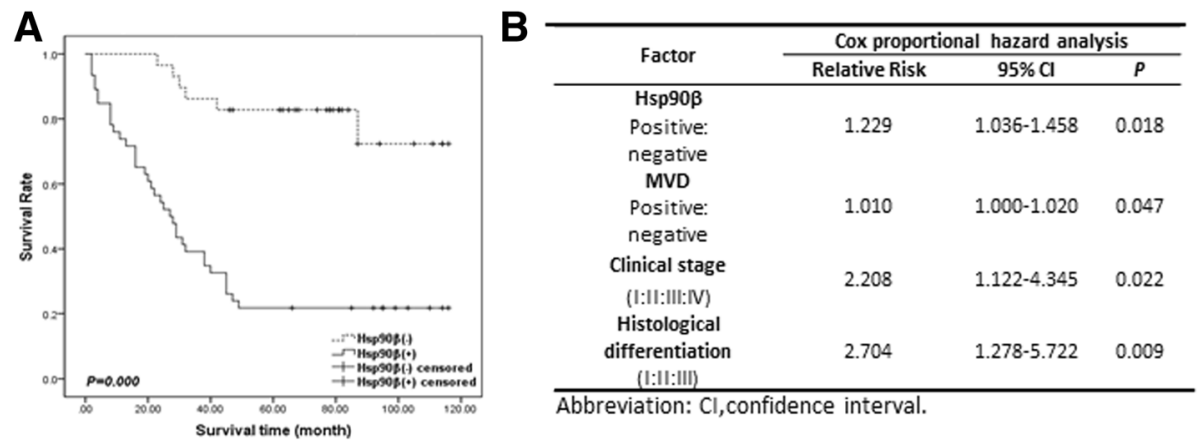

Fig. 2 Relationship between Hsp90ß and prognosis. a Kaplan-Meier curves for the OS of HCC patients with Hsp90ß-positive and Hsp90ß-negative expression. The Hsp90ß-negative group tended to have a longer survival time than the Hsp90ß-positive group $(P=0.027)$. b Multivariate analyses of factors influencing survival. Cox proportional hazard model analysis was performed with clinicopathological factors (clinical stage, $P=0.022$; histological differentiation, $P=0.009$ ), Hsp90 $\beta$ expression (positive/negative, $P=0.018$ ), and MVD (positive/negative, $P=0.047$ )

compared with alone Hsp90 $\beta$ knocked down group (Fig. 4a). Furthermore, we used a dual-luciferase report system to determine the effect of Hsp90 $\beta$ on the promoter activities of VEGFR1 and VEGFR2. The results showed that Hsp90 $\beta$ and/or VEGF increased VEGFR1 and VEGFR2 promoter activities, Hsp90 $\beta$ knockdown decreased VEGFR1 and VEGFR2 promoter activities and VEGF released the inhibition effect of Hsp90 $\beta$ knocked down on VEGFRs promoter activities (Fig. 4c). In addition, Hsp90 $\beta$ promoted HUVEC proliferation and Hsp90 $\beta$ knockdown inhibited HUVEC proliferation. VEGF treatment counteracted the inhibitory effect of knocked down Hsp90 $\beta$ (Fig. 4d). Then, we performed Transwell chamber (with or without Matrigel on the filters) invasion and migration assays to examine the effects of Hsp $90 \beta$ on HUVEC invasion and migration. The number of HUVECs that migrated through the filters showed that cell invasion and migration significantly increased after Hsp90 $\beta$ overexpression and/or VEGF treatment. The cell invasion and migration decreased obviously when Hsp90 $\beta$ knocked down and VEGF released the inhibition effect of knocked down Hsp90 $\beta$ (Fig. 4e and f).

In vitro, endothelial cells can form a three-dimensional tube in Matrigel culture. To examine the effects of Hsp90 $\beta$ on HUVEC tube formation, we seeded HUVECs that were transfected with Hsp90 $\beta$, Hsp90 $\beta$ siRNA, and/ or treated with VEGF for $48 \mathrm{~h}$ on Matrigel. The results showed that Hsp90 $\beta$ significantly enhanced HUVEC tube formation, whereas Hsp90 $\beta$ silencing inhibited HUVEC tube formation. VEGF increased the effect of Hsp90 $\beta$ on angiogenesis and knockdown of Hsp90 $\beta$ obviously decreased VEGF-induced tube formation (Fig. 4g). Our results showed that Hsp90 $\beta$ affected in vitro VEGFRs promoter activities, VEGFRs expression, and HUVEC proliferation, migration, invasion, tube formation, and angiogenesis.

Table 1 Correlation between Hsp90ß, VEGFR1, VEGFR2, and primary tumor characteristics

\begin{tabular}{|c|c|c|c|c|c|c|c|c|c|c|c|c|}
\hline \multirow[t]{2}{*}{ Variant } & \multicolumn{2}{|c|}{ Hsp90ß } & \multirow[t]{2}{*}{$x^{2}$} & \multirow[t]{2}{*}{$P$} & \multicolumn{2}{|c|}{ VEGFR1 } & \multirow[t]{2}{*}{$x^{2}$} & \multirow[t]{2}{*}{$P$} & \multicolumn{2}{|c|}{ VEGFR2 } & \multirow[t]{2}{*}{$x^{2}$} & \multirow[t]{2}{*}{$P$} \\
\hline & Low & High & & & Low & High & & & Low & High & & \\
\hline \multicolumn{13}{|c|}{ Age (year) } \\
\hline$\leq 60$ & 204 & 53 & 0.001 & 0.973 & 67 & 12 & 0.701 & 0.403 & 60 & 19 & 0.918 & 0.338 \\
\hline$>60$ & 39 & 10 & & & 13 & 4 & & & 11 & 6 & & \\
\hline \multicolumn{13}{|l|}{ Sex } \\
\hline M & 202 & 58 & 3.128 & 0.077 & 74 & 13 & 1.986 & 0.159 & 64 & 23 & 0.075 & 0.784 \\
\hline $\mathrm{F}$ & 41 & 5 & & & 6 & 3 & & & 7 & 2 & & \\
\hline \multicolumn{13}{|c|}{ Histological differentiation } \\
\hline$\leq \|$ & 180 & 38 & 4.621 & $0.032^{*}$ & 62 & 16 & 4.431 & $0.035^{*}$ & 61 & 17 & 3.895 & $0.048^{*}$ \\
\hline$>\| 1$ & 63 & 25 & & & 18 & 0 & & & 10 & 8 & & \\
\hline \multicolumn{13}{|l|}{ Stage } \\
\hline$\leq \|$ & 148 & 47 & 4.061 & $0.044^{*}$ & 46 & 14 & 5.120 & $0.024^{*}$ & 48 & 12 & 3.032 & 0.082 \\
\hline$>\| 1$ & 95 & 16 & & & 34 & 2 & & & 23 & 13 & & \\
\hline
\end{tabular}

*Significantly different, $P<0.05$ 

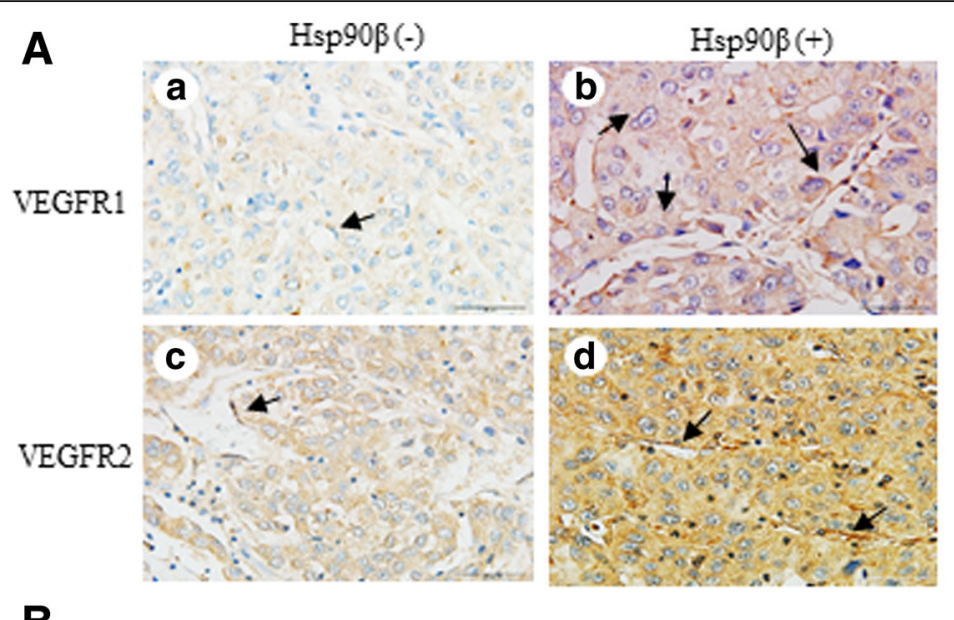

B
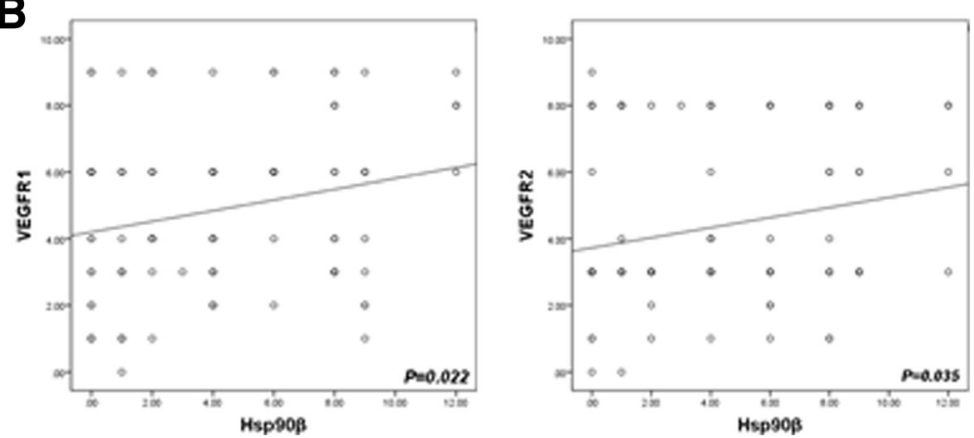

Fig. 3 VEGFR1 and VEGFR2 expression in Hsp90ß-negative (Hsp90ß-) and Hsp90ß-positive (Hsp90ß+) HCC tissues. a $a$ and C, VEGFR1 and VEGFR2 expression in Hsp90ß- HHC group; $b$ and d, VEGFR1 and VEGFR2 expression in Hsp90ß + HCC group (original magnification $\times 400$ ). Black arrows point to microvessels. $\mathbf{b}$ Correlation analysis between Hsp90 $\beta$ and VEGFR1 $(P=0.022)$ and VEGFR2 $(P=0.035)$

Hsp90 inhibitor blocked the proliferation, migration, invasion, and tube formation of endothelial cells

The ability of Hsp90 inhibitors, such as geldanamycin and its derivatives, to suppress endothelial cell proliferation has been previously described [26]. However, the effect of NVP-BEP800, a new Hsp90 inhibitor, on endothelial cells has not been reported. To determine the direct cytotoxic effects of NVP-BEP800 on endothelial cells, we conducted an in vitro cytotoxicity experiment. The different concentrations of NVP-BEP800 were cytotoxic to HUVECs when given as a treatment for $48 \mathrm{~h}$ (Fig. 5a). In addition, the toxicity of NVP-BEP800 in normal human liver cells (HHL-5) and various HCC cell lines (SMMC-7721, PLC-PRF-5, BEL-7402) were also measured. The results showed that NVP-BEP800 has no significant toxicity in HHL-5, and inhibited the proliferation of HCCs (Additional file 2 Fig. S2). HUVECs in logphase growth were measured over $72 \mathrm{~h}$ and showed that NVP-BEP800 $(2 \mu \mathrm{M})$ inhibited the proliferation of human endothelial cells (Fig. 5b). There was no obvious effect on death and apoptosis of HUVECs under $2 \mu \mathrm{M}$ NVP-BEP800 treatment for $48 \mathrm{~h}$ (Additional file 3 Fig. S3). Western blot analysis showed that VEGFR1 and VEGFR2 expressions decreased under $48 \mathrm{~h}$ of $2 \mu \mathrm{M}$ NVP-
BEP800 treatment (Fig. 5c). Furthermore, we analyzed the effect of NVP-BEP800 on VEGFRs promoter activities. The results of the dual-luciferase reporter assay showed that NVP-BEP800 significantly decreased VEGFR1 and VEGFR2 promoter activities (Fig. 5d). In addition, a scratch wound assay showed that Hsp90 inhibitors prevented HUVEC haptotaxis. Control cells completed wound closure after $48 \mathrm{~h}$. By contrast, cells treated with $2 \mu \mathrm{M}$ NVP-BEP800 failed to complete wound closure within $48 \mathrm{~h}$ (Fig. 5e). To test the effect of NVP-BEP800 inhibition on HUVEC tube formation, HUVECs were treated with $2 \mu \mathrm{M}$ NVP-BEP800 for $24 \mathrm{~h}$ and then seeded on Matrigel for $12 \mathrm{~h}$. The results showed that NVPBEP800 significantly decreased tube formation compared with control cells (Fig. 5f). We evaluated the tube formation in HUVECs that knocked down Hsp90 $\alpha$ or Hsp90 $\beta$ under NVP-BEP800 treatment. The results showed that compared to Hsp90 $\alpha$ knocked down group, the tube formation in siHsp90 $\alpha+$ NVP-BEP800 group were significantly reduced. While there was no statistically significant difference in Hsp90 $\beta$ knocked down group and siHsp90 $\beta+$ NVP-BEP800 group (Additonal file 4 Fig. S4). It is indicated that the inhibition of endothelial celldependent tumor angiogenesis of NVP-BEP800 was 

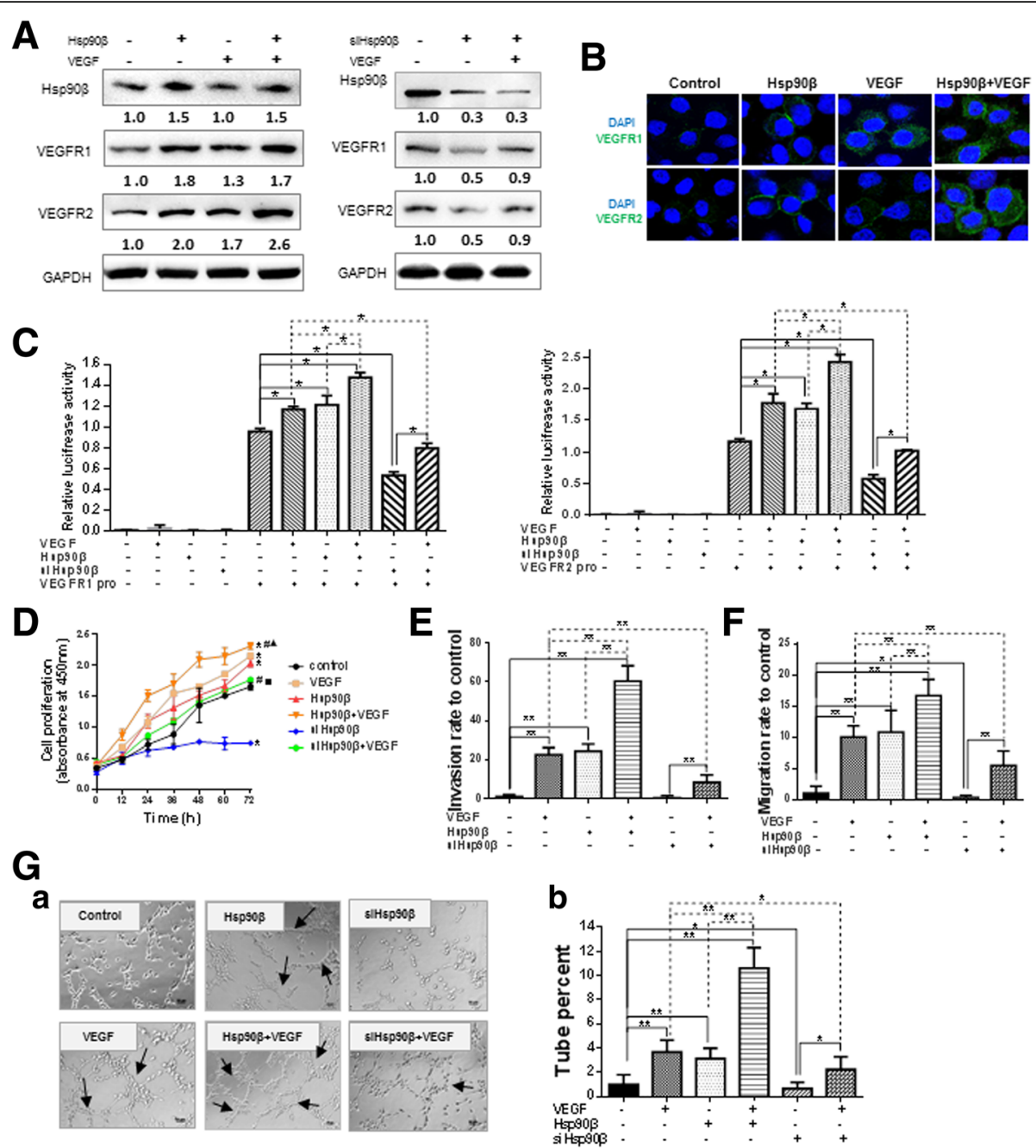

Fig. $4 \mathrm{Hsp90 \beta}$ promotes endotheliocyte proliferation and accelerates neovascularization. a Western blot analysis showed VEGFR1 and VEGFR2 expression levels in HUVEC cells overexpressed or knocked down Hsp90ß and/or under VEGF treatment. $\mathbf{b}$ Immunofluorescence of VEGFR1 and VEGFR2 expression in HUVEC cells overexpressed Hsp90ß alone, VEGF treatment alone or overexpressed Hsp90ß under VEGF treatment. c HUVEC cells were transiently transfected with VEGFR1- or VEGFR2- dependent reporter gene plasmids. Luciferase activity was measured when cells overexpressed or knocked down Hsp90ß and/or under VEGF treatment. d Growth curve of HUVEC cells overexpressed or knocked down Hsp90ß and/or under VEGF treatment, measured by SRB assay. ( ${ }^{*}$, vs control group, $P<0.05$; \#, vs VEGF group, $P<0.05$; $\mathbf{\Lambda}$, vs Hsp90ß group, $P<0.05$; $\mathbf{\square}$, vs siHsp90 $\beta$ group, $P<0.05)$ e Transwell assays indicated invasion ability when HUVEC cells overexpressed or knocked down Hsp90 $\beta$ and/or under VEGF treatment. $\mathbf{f}$ Migration ability increased when HUVEC cells overexpressed or knocked down Hsp90ß and/or under VEGF treatment. $\mathbf{g}$ In vitro assay for vascular mimicry of HUVEC cells in three-dimensional culture at $12 \mathrm{~h}$. $a$, Representative graphs; black arrows point to tubes. $b$, Tube formation quantification was analyzed after HUVEC cells overexpressed or knocked down Hsp90ß and/or under VEGF treatment. ${ }^{*} P<0.05,{ }^{* *} P<0.01$

primarily by inhibiting the activity of $\mathrm{Hsp} 90 \mathrm{\beta}$. In addition, 17-Allylamino-17-demethoxygeldanamycin (17-AAG) were compared with NVP BEP800 on the proliferation and tube formation of HUVECs (Additonal file 5 Fig. S5). The results showed that 17-AAG inhibited cell proliferation, while the inhibition effect of NVP-BEP800 was stronger than 17-AAG on tube formation.

\section{Hsp90ß promoted angiogenesis and Hsp90 inhibitor suppressed angiogenesis in vivo}

We demonstrated that Hsp90 $\beta$ promoted endothelial cell proliferation, migration, and tube formation and that the Hsp90 inhibitor attenuated VEGFR levels and inhibited tube formation in vitro. Then, we constructed a nude mice subskin model that was transplanted with human liver cancer. Control group mice subcutaneously received PLC cell suspensions. Hsp90 $\beta$ group mice subcutaneously received a suspension of PLC cells transfected with pcDNA-Hsp90 $\beta$ plasmids. NVP-BEP800 group mice subcutaneously received a suspension of PLC cells. When the tumors volume reached 100$200 \mathrm{~mm}^{3}$, NVP-BEP800 group mice were treated with NVP-BEP800 (30 mg/kg/daily) by intragastric administration for 18 days and solvent buffer at the same 


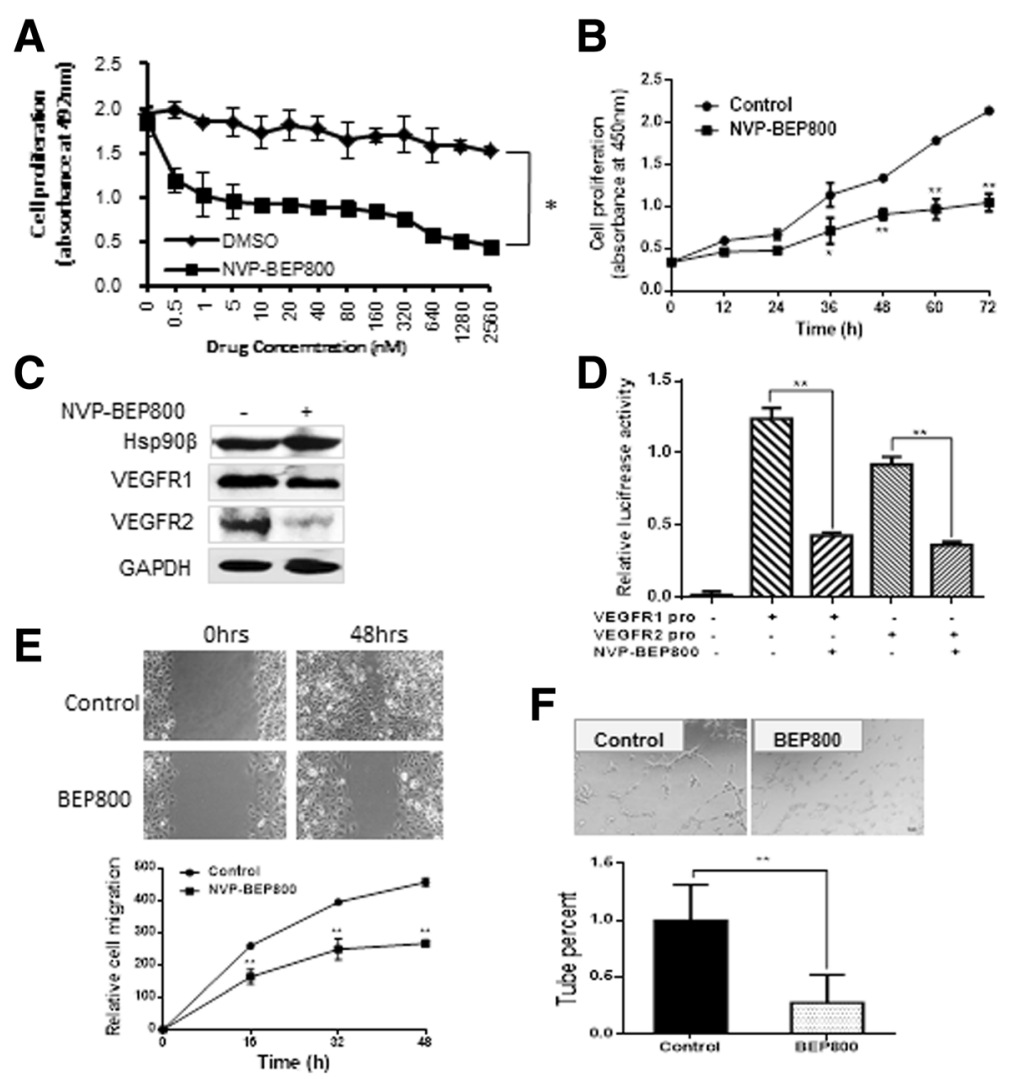

Fig. 5 NVP-BEP800 inhibited endothelial cell proliferation, migration, invasion, and tube formation. a Incubation with different concentrations of NVP-BEP800 for $48 \mathrm{~h}$ inhibited HUVEC cell proliferation. b Growth curve of HUVEC cells incubated with $2 \mu$ M NVP-BEP800, as measured by SRB assay. $\mathbf{c}$ Western blot analysis showed that treatment with $2 \mu \mathrm{M}$ NVP-BEP800 down-regulated VEGFR1 and VEGFR2 expression in HUVEC cells. $\mathbf{d}$ HUVEC cells were transiently transfected with VEGFR1- or VEGFR2- dependent reporter gene plasmids and treated with $2 \mu \mathrm{M}$ NVP-BEP800. After $24 \mathrm{~h}$, the cells were lysed and luciferase activity was measured. e The wound healing ability of HUVEC cells was assessed after $48 \mathrm{~h}$ of NVP-BEP800 treatment. DMSO treatment was used as the standardized control for quantification. $\mathbf{f}$ In vitro assay for vascular mimicry of HUVEC cells treated with NVP-BEP800 for $48 \mathrm{~h}$ in three-dimensional culture. NVP-BEP800 inhibited cord formation of HUVEC cells. ${ }^{*} P<0.05,{ }^{* *} P<0.01$

volume was used in control and Hsp90 $\beta$ groups. The results showed that compared with the control group, Hsp90 $\beta$ promoted the liver tumor growth, and NVPBEP800 inhibited tumor growth in vivo (Fig. 6a, b). To further check the effect of Hsp90 $\beta$ and NVP-BEP800 on cell proliferation, immunohistochemical analysis of Ki67 was performed with the tumor issues (Fig. c, d). Also, we demonstrated that VGFR1 and VEGFR2 expression levels increased in cells overexpressing Hsp90 $\beta$, but decreased after NVP-BEP800 treatment (Fig. 6e, f). Immunohistochemical detection revealed that CD31 expression and MVD in the Hsp90 $\beta$-overexpressing group were significantly higher than those in the control group, but decreased in the presence of NVP-BEP800 (Fig. 6g, h). There was no significant difference about blood vessel tissue between control and NVP-BEP800 group by analysis mice liver and lung pathological section using hematoxylin and eosin staining (Additonal file 6 Fig. S6). Our data suggested that Hsp90 $\beta$ contributed to endothelial cell-dependent angiogenesis.

\section{Discussion}

Angiogenesis is critical to tumor growth, invasion, and metastasis, especially during the early stages of tumor development. Our previous work demonstrated that Hsp90 $\beta$ is a marker protein for detecting liver cancer malignancy. Based on the analysis of 96 liver cancer cases, we demonstrated that Hsp90 $\beta$ was up-regulated in HCCs with a high degree of malignancy. Especially, in HCC tissues, Hsp90 $\beta$ expression in vascular endothelial cells also significantly increased. We examined CD31positive endothelial cell-dependent vascular density and found that Hsp90 $\beta$ expression was positively correlated with MVD. The survival time of Hsp90 $\beta$-negative HCC patients was longer than that of Hsp90 $\beta$-positive HCC patients. Cox regression analysis showed that Hsp90 $\beta$ expression and MVD, as well as the clinical stage and pathological grade of the tumor, are risk factors that determine the survival of HCC patients. Furthermore, we found that VEGFRs were highly expressed in HCC tissues with high Hsp90 $\beta$ expression in endothelial cells. 

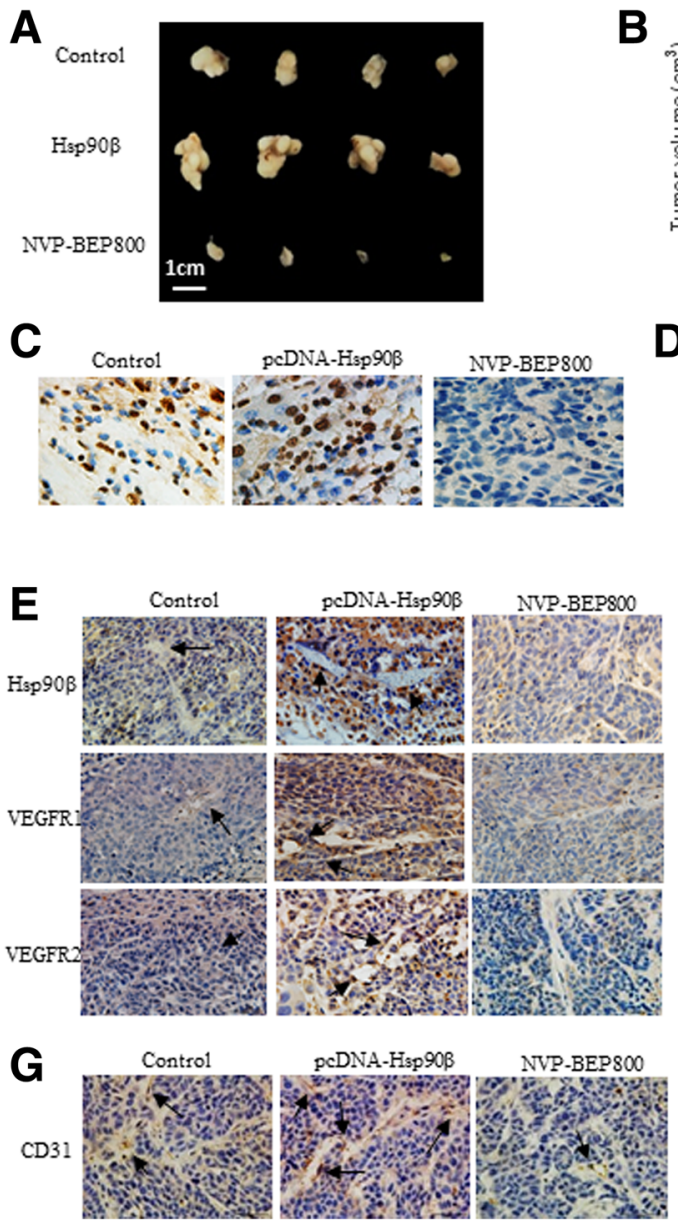

B
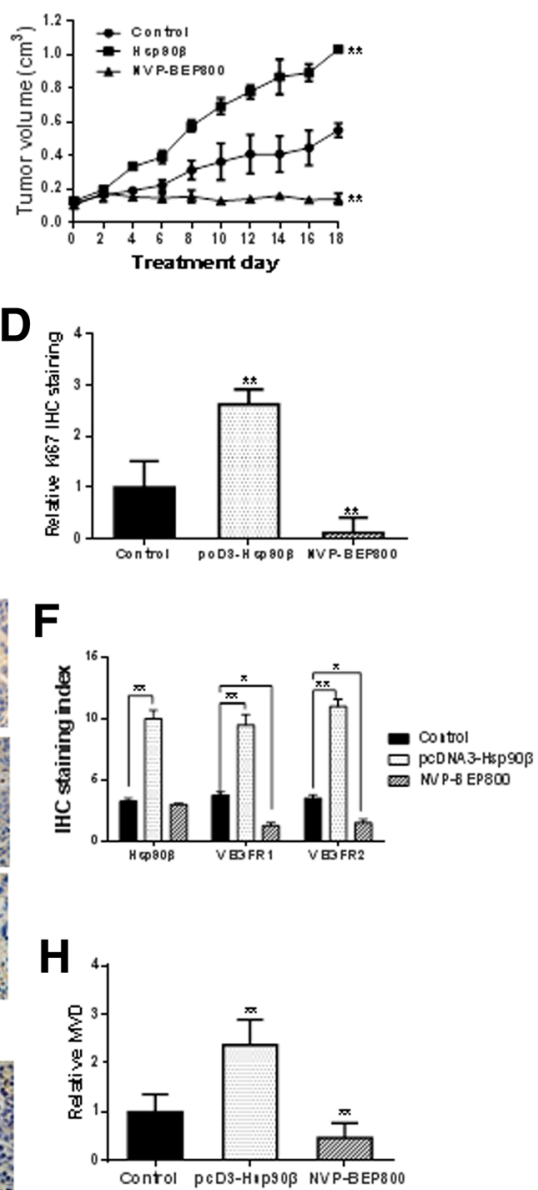

Fig. 6 Immunohistochemical analysis of VEGFRs expression and tumor vascularization in a HCC xenograft model. a Control group mice subcutaneously received PLC cell suspensions. Hsp90ß group mice subcutaneously received a suspension of PLC cells transfected with pCDNA-Hsp90 3 plasmids. NVP-BEP800 group mice subcutaneously received a suspension of PLC cells and were treated by intragastric administration with NVP-BEP800 (30 mg/kg/day). b Tumor sizes were measured every 2 days and tumor volumes were calculated. $\mathbf{c}$, $\mathbf{d}$ To check the effect of Hsp90 3 and NVP-BEP800 on cell proliferation, immunohistochemical analysis of Ki67 was performed with the tumor issues. The intensity of Ki67-positive cell was calculated with Image J software. e, f VEGFR1 and VEGFR2 expression in tumor tissue of the control group, Hsp90 3 group, and NVP-BEP800 group mice. $\mathbf{g}$, $\mathbf{h}$ Analysis of MVD based on CD31 staining of tumor tissue by Image J software showed that vessel number significantly increased in the Hsp90 $\beta$ group and that vessel number decreased in the NVP-BEP800 group. ${ }^{*} P<0.05$, ${ }^{*} P<0.01$

Statistical analysis showed that Hsp90ß expression was correlated with VEGFRs.

In vitro, Hsp90 $\beta$ promoted VEGFR1 and VEGFR2 promoter activities. Furthermore, Hsp $90 \beta$ promoted VEGFR expression in endothelial cells. VEGFRs are crucial to tumor angiogenesis, promote the proliferation of endothelial cells, and increase vascular permeability [27]. The stimulating effect of $\mathrm{Hsp} 90 \beta$ was more obvious on VEGFR2 promoter activity than on VEGFR1 promoter activity, which indicated that Hsp90 $\beta$ mainly acts on VEGFR2. After Hsp90 $\beta$ overexpression, endothelial cell proliferation, invasion, migration, and tubular structure formation increased. The promoting effect was more apparent when VEGF treatment was simultaneously performed. This may be due to Hsp90 $\beta$ promoting VEGFRs expression by stimulating VEGFRs promoter activities, and after VEGF treatment, more receptors were induced to combine with VEGF and lead to active a series of downstream pathways. Hsp90 $\beta$ knockdown decreased the ability of endothelial cells to form tube structures. Tube formation of simultaneous Hsp90 $\beta$ knockdown and VEGF treatment group was decreased obviously compared with the VEGF-only treated group. This finding indicated that knocked-down Hsp90 $\beta$ counteracted the tube formation induced by VEGF. Compared with $\mathrm{Hsp} 90 \beta$ knockdown group, the simultaneous Hsp90 $\beta$ knockdown and VEGF treatment group had increased tube formation. This finding indicated that VEGF can release the inhibition caused by Hsp90 $\beta$ knockdown in HUVEC cells. Also, it means that VEGF can release the inhibition on 
angiogenesis caused by Hsp90 $\beta$ knockdown. Intratumoral MVD and VEGFRs expression increased in the nude mice that were subcutaneously tumor burdened with Hsp90ßoverexpressing HCC cells.

Based on the role of $\mathrm{Hsp} 90 \mathrm{\beta}$ in angiogenesis, we sought to identify Hsp90 $\beta$ inhibitors as antitumor agents by anti-angiogenesis. Hsp90 inhibitors, such as NVPAUY922 [28], VER49009/VER50589 [29], and geldanamycin derivatives, including 17-AAG and 17-DMAG $[26,30-32]$, are used as antitumor and antiangiogenesis drugs. The direct effect of NVP-BEP800, a new Hsp90 $\beta$ inhibitor, on anti endothelial cell-dependent angiogenesis has never been reported. The antiangiogenic activities of Hsp90 $\beta$ inhibitors directly affected endothelial cells. NVPBEP800 inhibited vascular endothelial cell proliferation. This inhibitory effect was dose-dependent. Endothelial cell migration, invasion, and tube formation directly affected the angiogenic process. There was reported that Hsp90 forms a complex with VEGFR2, but VEGFR2 deletion mutants cannot associate with Hsp90 [19, 25]. Interestingly, our findings showed that NVP-BEP800 decreased VEGFR1 and VEGFR2 expression levels by inhibiting VEGFR1 and VEGFR2 promoter activities. Our in vitro studies indicated that tumors in mice treated with NVP-BEP800 showed decreased VEGFRs expression and reduced microvascular density. In addition, no significant difference in the blood vessel tissues of liver and lung was observed between the normal and NVP-BEP800 treated mice. So we hold that the NVP-BEP800 may only affect the tumor neovascularization process and may have no significant effect on the blood vessels of the normal tissues.

In conclusion, our data illustrated that $\mathrm{Hsp} 90 \beta$ promoted endothelial cell-dependent tumor angiogenesis by increasing VEGFRs expression. Moreover, NVP-BEP800, a Hsp90 inhibitor, exerted antiangiogenic activity when administered orally. Moreover, it is reported that NVP-BEP800 used as an antineoplastic agent, via decreasing Hsp90 client proteins of downstream [22]. Therefore, Hsp90 inhibitors may target multiple pivotal points in both tumor and endothelial cell survival, proliferation, and invasion to provide a combinatorial approach to attack multiple features of malignant phenotypes [18]. Hsp90 inhibitors may be used as a therapeutic strategy to inhibit tumor angiogenesis by decreasing endothelial cell progression and metastasis.

\section{Conclusions}

Hsp90 $\beta$ induced endothelial cell-dependent tumor angiogenesis by activating VEGFRs transcription. We provided new insight into the regulatory mechanism of tumor angiogenesis by exploring the role of $\mathrm{Hsp} 90 \beta$, which may also help develop a therapeutic strategy for tumor treatment through the inhibition of endothelial cell progression and metastasis.

\section{Additional files}

Additional file 1: Figure S1. Comparative distribution of the Hsp90 $\beta$ and CD31 (a), VEGFR1 (b), VEGFR2 (c) expression levels. (TIF 438 kb)

Additional file 2: Figure S2. Inhibition of HHL-5 (a), SMMC-7721 (b), PLCPRF-5 (c), and BEL-7402 (d) cells via NVP-BEP800 treatment. (TIF 647 kb)

Additional file 3: Figure S3. HUVEC cell death and apoptosis results under NVP-BEP800 treatment. (TIF $1300 \mathrm{~kb}$ )

Additional file 4: Figure S4. (a) Western blot analysis of Hsp90a and Hsp90 $\beta$ expression in HUVEC cells with knocked down Hsp90a or Hsp90ß under NVP-BEP800 treatment. (b) Tube. (TIF 1105 kb)

Additional file 5: Figure S5. Inhibition of HUVEC cell proliferation (a) and tube formation (b) via 17-AAG and NVP-BEP800 treatments. (TIF 824 kb)

Additional file 6: Figure S6. HE staining of mice liver and lung pathological sections in the control and NVP-BEP800 groups. (TIF 4229 kb)

\section{Abbreviations}

HCC: Hepatocellular carcinoma; Hsp90ß: Heat shock protein 90beta; MVD: Microvascular density; SEAP: Secreted alkaline phosphatase; VEGFR: Vascular endothelial growth factor receptor

\section{Acknowledgements}

We thank Tianjin International Joint Academy of Biomedicine to provide animal feeding room.

\section{Funding}

This study was supported by the National Natural Science Funds of China [Grants 81402973; 81402973]; the Key Technologies R\&D Program of Tianjin [Grant 11ZCKFSY06900]; Tianjin Natural Science and Technology Found [Grant 15JCYBJC26400]; and Foundation for the Author of National Excellent Doctoral Dissertation of China [Grant 201482]; Natural Science Foundation of Tianjin [Grant 16JCQNJC13300]. Innovation fund for technology based firms [12ZXCXSY06500; 12ZXCXSY07200].

\section{Availability of data and materials}

The datasets analyzed during the current study is available from the corresponding author on reasonable request.

\section{Authors' contributions}

$J M, T S$ and $C Y$ developed the original hypothesis and experimental design. $J M, J H$ and $Q T$ performed in vitro experiments. JM, YL carried out animal studies. SC and KQ did the immunochemical staining. JM, YL, TS, HZ acquired and analyzed data. JM wrote the manuscript. All read and approved the final manuscript.

\section{Competing interests}

The authors declare that they have no competing interests.

Consent for publication

Not applicable.

Ethics approval and consent to participate

Not applicable.

\section{Publisher's Note}

Springer Nature remains neutral with regard to jurisdictional claims in published maps and institutional affiliations.

\section{Author details}

${ }^{1}$ State Key Laboratory of Medicinal Chemical Biology and College of Pharmacy, Nankai University, Tianjin 300350, China. ${ }^{2}$ Tianjin Key Laboratory of Molecular Drug Research, Tianjin International Joint Academy of Biomedicine, Tianjin, China. 
Received: 7 December 2016 Accepted: 19 March 2017

Published online: 31 March 2017

\section{References}

1. Park JH, Kim SH, Choi MC, Lee J, Oh DY, Im SA, Bang YJ, et al. Class ॥ histone deacetylases play pivotal roles in heat shock protein 90-mediated proteasomal degradation of vascular endothelial growth factor receptors. Biochem Biophys Res Commun. 2008;368:318-22.

2. Zhao Y, Adjei AA. Targeting Angiogenesis in Cancer Therapy: Moving Beyond Vascular Endothelial Growth Factor. Oncologist. 2015;20:660-73.

3. Sun J, Liao JK. Induction of angiogenesis by heat shock protein 90 mediated by protein kinase Akt and endothelial nitric oxide synthase. Arterioscler Thromb Vasc Biol. 2004;24:2238-44.

4. Carmeliet $P$, Jain RK. Angiogenesis in cancer and other diseases. Nature. 2000:407:249-57.

5. Staufer K, Stoeltzing O. Implication of heat shock protein 90 (HSP90) in tumor angiogenesis: a molecular target for anti-angiogenic therapy? Curr Cancer Drug Targets. 2010;10:890-7.

6. Shibuya M. Vascular endothelial growth factor receptor-1 (VEGFR-1/FIt-1): a dual regulator for angiogenesis. Angiogenesis. 2006;9:225-30. discussion 231.

7. Ferrara N, Davis-Smyth T. The biology of vascular endothelial growth factor. Endocr Rev. 1997:18:4-25.

8. Rapisarda A, Melillo G. Role of the VEGFNEGFR axis in cancer biology and therapy. Adv Cancer Res. 2012;114:237-67.

9. Karkkainen MJ, Petrova TV. Vascular endothelial growth factor receptors in the regulation of angiogenesis and lymphangiogenesis. Oncogene. 2000;19: 5598-605.

10. Koch S, Claesson-Welsh L. Signal transduction by vascular endothelial growth factor receptors. Cold Spring Harb Perspect Med. 2012;2:a006502.

11. Cross MJ, Dixelius J, Matsumoto T, Claesson-Welsh L. VEGF-receptor signal transduction. Trends Biochem Sci. 2003;28:488-94.

12. Zachary I. VEGF signalling: integration and multi-tasking in endothelial cell biology. Biochem Soc Trans. 2003;31:1171-7.

13. Rundhaug JE. Matrix metalloproteinases and angiogenesis. J Cell Mol Med. 2005:9:267-85

14. Deryugina El, Quigley JP. Tumor angiogenesis: MMP-mediated induction of intravasation- and metastasis-sustaining neovasculature. Matrix Biol. 2015;46:94-112

15. Neckers L. Heat shock protein 90: the cancer chaperone. J Biosci. 2007;32:517-30

16. Xue Q, Nagy JA, Manseau EJ, Phung TL, Dvorak HF, Benjamin LE. Rapamycin inhibition of the Akt/mTOR pathway blocks select stages of VEGF-A164driven angiogenesis, in part by blocking S6Kinase. Arterioscler Thromb Vasc Biol. 2009:29:1172-8

17. Saryeddine L, Zibara K, Kassem N, Badran B, El-Zein N. EGF-Induced VEGF Exerts a PI3K-Dependent Positive Feedback on ERK and AKT through VEGFR2 in Hematological In Vitro Models. PLOS ONE. 2016;11:e0165876.

18. Sanderson S, Valenti M, Gowan S, Patterson L, Ahmad Z, Workman P, Eccles SA. Benzoquinone ansamycin heat shock protein 90 inhibitors modulate multiple functions required for tumor angiogenesis. Mol Cancer Ther. 2006; 5:522-32.

19. Masson-Gadais B, Houle F, Laferriere J, Huot J. Integrin alphavbeta3, requirement for VEGFR2-mediated activation of SAPK2/p38 and for Hsp90dependent phosphorylation of focal adhesion kinase in endothelial cells activated by VEGF. Cell Stress Chaperones. 2003;8:37-52.

20. Sampat KR, O'Neil B. Antiangiogenic therapies for advanced hepatocellular carcinoma. Oncologist. 2013;18:430-8

21. Kim HY, Kim J, Ha Thi HT, Bang OS, Lee WS, Hong S. Evaluation of antitumorigenic activity of BP3B against colon cancer with patient-derived tumor xenograft model. BMC Complement Altern Med. 2016:16:473.

22. Massey AJ, Schoepfer J, Brough PA, Brueggen J, Chene P, Drysdale MJ, Pfaar U, et al. Preclinical antitumor activity of the orally available heat shock protein 90 inhibitor NVP-BEP800. Mol Cancer Ther. 2010:9:906-19.

23. Zhang D, Hedlund EM, Lim S, Chen F, Zhang Y, Sun B, Cao Y. Antiangiogenic agents significantly improve survival in tumor-bearing mice by increasing tolerance to chemotherapy-induced toxicity. Proc Natl Acad Sci U S A. 2011:108:4117-22.

24. Shibuya M. Vascular Endothelial Growth Factor (VEGF) and Its Receptor (VEGFR) Signaling in Angiogenesis: A Crucial Target for Anti- and ProAngiogenic Therapies. Genes Cancer. 2011;2:1097-105.
25. Le Boeuf F, Houle F, Huot J. Regulation of vascular endothelial growth factor receptor 2-mediated phosphorylation of focal adhesion kinase by heat shock protein 90 and Src kinase activities. J Biol Chem. 2004;279: 39175-85.

26. Kaur G, Belotti D, Burger AM, Fisher-Nielson K, Borsotti P, Riccardi E, Thillainathan J, et al. Antiangiogenic properties of 17-(dimethylaminoethylamino)-17demethoxygeldanamycin: an orally bioavailable heat shock protein 90 modulator. Clin Cancer Res. 2004;10:4813-21.

27. Tabernero J. The role of VEGF and EGFR inhibition: implications for combining anti-VEGF and anti-EGFR agents. Mol Cancer Res. 2007;5:203-20.

28. Eccles SA, Massey A, Raynaud Fl, Sharp SY, Box G, Valenti M, Patterson L, et al. NVP-AUY922: a novel heat shock protein 90 inhibitor active against xenograft tumor growth, angiogenesis, and metastasis. Cancer Res. 2008;68: 2850-60.

29. Sharp SY, Prodromou C, Boxall K, Powers MV, Holmes JL, Box G, Matthews TP, et al. Inhibition of the heat shock protein 90 molecular chaperone in vitro and in vivo by novel, synthetic, potent resorcinylic pyrazole/isoxazole amide analogues. Mol Cancer Ther. 2007;6:1198-211.

30. Burger AM, Fiebig HH, Stinson SF, Sausville EA. 17-(Allylamino)-17demethoxygeldanamycin activity in human melanoma models. Anticancer Drugs. 2004;15:377-87.

31. Kim WY, Oh SH, Woo JK, Hong WK, Lee HY. Targeting heat shock protein 90 overrides the resistance of lung cancer cells by blocking radiation-induced stabilization of hypoxia-inducible factor-1alpha. Cancer Res. 2009:69:1624-32.

32. Lang SA, Klein D, Moser C, Gaumann A, Glockzin G, Dahlke MH, Dietmaier W, et al. Inhibition of heat shock protein 90 impairs epidermal growth factormediated signaling in gastric cancer cells and reduces tumor growth and vascularization in vivo. Mol Cancer Ther. 2007:6:1123-32.

\section{Submit your next manuscript to BioMed Central and we will help you at every step:}

- We accept pre-submission inquiries

- Our selector tool helps you to find the most relevant journal

- We provide round the clock customer support

- Convenient online submission

- Thorough peer review

- Inclusion in PubMed and all major indexing services

- Maximum visibility for your research

Submit your manuscript at www.biomedcentral.com/submit
C) BioMed Central 